\title{
Deletion 9p23 to 9p11.1 as sole additional abnormality in a Philadelphia positive chronic myeloid leukemia in blast crisis: a rare event
}

\author{
Abdulsamad Wafa', Manar Asa'ad', Adnan Ikhtiar², Thomas Liehr ${ }^{3}$ and Walid Al-Achkar ${ }^{1 *}$
}

\begin{abstract}
Background: Chronic myeloid leukemia (CML) is a myeloproliferative disorder characterized by the presence of a derivative chromosome 22 [der(22)] commonly called Philadelphia chromosome (Ph). The Ph chromosome is a product of the reciprocal translocation $t(9 ; 22)(q 34.1 ; q 11.2)$. Additional genetic changes occur in less than $10 \%$ of CML cases at the time of diagnosis and other genetic changes are seen in 60-80\% of the cases in advanced disease. Even though deletions in chromosome 9 are not rare findings in advanced phase-CML, del(9)(p23p11.1) as sole additional abnormality detected by fluorescence in situ hybridization (FISH) technique, to our knowledge has not been described in the literature.

Results: A complete cytogenetic and molecular cytogenetic analysis, molecular biology method (reverse transcription polymerase chain reaction (RT-PCR)), and immunophenotype confirmed to be a CML case in blast crisis (BC). It revealed del(9)(p23p11.1) as sole abnormality detected by FISH technique besides Ph chromosome, which leads to monoallely of tumor suppressor gene CDKN2A (cyclin-dependent kinase inhibitor 2A) before Imatinib mesylate (IM) treatment.
\end{abstract}

Conclusions: The patient did not demonstrate a good response to IM treatment. The underlying mechanisms and prognostic implications of these cytogenetic abnormalities are discussed.

Keywords: Chronic myeloid leukemia, Philadelphia chromosome, del(9)(p24p12), CDKN2A gene, Prognostic factors

\section{Background}

Chronic myeloid leukemia (CML) is a myeloproliferative disorder. It is mainly characterized by the presence of a derivative chromosome 22 [der(22)], the so-called Philadelphia chromosome $(\mathrm{Ph})$, which is due to a reciprocal translocation $\mathrm{t}(9 ; 22)(\mathrm{q} 34.1 ; \mathrm{q} 11.2)$. This rearrangement involves the breakpoint cluster region $(B C R)$ gene 22q11.2 and the c-Abelson (ABL1) gene 9q34, resulting in the BCR-ABL1 fusion gene, encoding a constitutively active tyrosine kinase protein [1].

The $\mathrm{Ph}$ chromosome can be observed as a sole chromosomal anomaly during the early chronic phase (CP) of CML (in more than $90 \%$ of the CML cases), additional genetic changes occur in less than $10 \%$ of cases at the time of diagnosis and other genetic changes

\footnotetext{
* Correspondence: ascientific@aec.org.sy

'Department of Molecular Biology and Biotechnology, Human Genetics

Division, Atomic Energy Commission, P.O. Box 6091, Damascus, Syria

Full list of author information is available at the end of the article
}

are seen in $60-80 \%$ of the cases in advanced disease [1]. The identification of this abnormality is important for the diagnosis of the disease determined by the WHO Tumor Classification [2] and for treatment purposes. The first therapeutic choice, tyrosine kinas inhibitors, has shown great therapeutic efficacy [3]. Imatinib mesylate (IM = Glivec, formerly STI571) is a chemically designed drug able to block BCR/ABL1 tyrosine kinase activity and is successfully used in CML patients [4].

Here we presented a de novo untreated $\mathrm{Ph}$-positive CML case in BC phase with del(9)(p23p11.1) as sole additional abnormality detected by FISH technique, which leads to monoallely of tumor suppressor gene CDKN2A (cyclin-dependent kinase inhibitor 2A). This patient did not demonstrate a good response to IM treatment.

\section{Case presentation}

In October 2013 a 40-year-old male was diagnosed as suffering from CML. Physical examination revealed the 
indicative symptoms as hepatosplenomegaly, loss of weight, fever, anemia, and thrombocytopenia. Routine peripheral blood test showed elevated white blood cells (WBC) of $243.1 \times 10^{9} / 1$ (41 \% of cells were blasts), red blood cell (RBC) count was $3.62 \times 10^{6} / \mathrm{mm}^{3}$, hemoglobin level was $6.8 \mathrm{~g} / \mathrm{dl}$ and the platelet count $26 \times 10^{9} / \mathrm{l}$. Serum lactate dehydrogenase value (LDH) was $1,543 \mathrm{U} / 1$ (normal level $<460 \mathrm{U} / \mathrm{l}$ ). The patient was diagnosed as CML-BC according to WHO recommendations, in a high Sokal risk of 29.7 (0.8-1.2), high Hasford (Euro) risk of 2,856 (>1,480), low Eutos risk of $40 \quad(<87)$ and Etous probability of no complete cytogenetic response (CCgR) at 18 months was $15 \%$. In fact, the patient started on IM treatment ( $400 \mathrm{mg} /$ day) for overall 1 month; then he increased IM daily dose to $800 \mathrm{mg}$ for 1 month but the treatment was not successful and the patient had a toothache, anemia, and thrombocytopenia. Later he was given IM $400 \mathrm{mg}$ daily dose for 2 months. Under the latter therapy the previous reported relevant symptoms disappeared. Unfortunately the patient died 7 months after diagnosis from the disease under the treatment for unknown reason.

\section{Results}

Prior to the IM-treatment GTG-banding revealed a karyotype of 46,XY,t(9;22)[11]/ 46,XY,t(9;22),del(9)(p?)[9]
(Fig. 1). Further molecular cytogenetics studies were performed (Fig. 2). Dual-color-fluorescence in situ hybridization (FISH) prior to IM-treatment using a specific probe for BCR and ABL1 revealed two fusion signals, on the der(9) and der(22), respectively (Fig. 2a). A probe-set specific for subtelomeric (ST) regions in $9 p$ and $9 \mathrm{q}$ revealed one green and one red signal on $\operatorname{der}(9)$, and one green, and one red signal on the $\operatorname{der}(9)$ and der(22), respectively (Fig. 2b). Chromosome 9 was studied with whole chromosome painting (WCP) probe and did not provide any hint on cryptic translocations (data not shown). A probe specific for $C D K N 2 A$ confirmed that the deletion visible in the short arm encompassed subband 9p21 (Fig. 2c). Multicolor banding (MCB) using a specific probe for chromosome 9 confirmed a terminal deletion of 9p23 to 9p11.1 (Fig. 2d). RT-PCR pre IM treatment confirmed the presence of the BCR-ABL1 fusion (b2a2 transcript) revealing a major $\mathrm{M}-\mathrm{BCR}$ transcript, most often identified in CML (data not shown). Thus, the following final karyotype prior IM-treatment was determined:

46,XY,t(9;22)(q34;q11.2)[11]/46,XY,der(9)(9pter- > 9p 23::9p11.1- > 9q34::22q11.2- > 22qter), der(22)t(9;22)(q34; q11.2)[9].

The abnormal cell population ( $\sim 22-45 \%$ blasts in peripheral blood specimen) showed the following immunophenotype, which was consistent with CML-BC (WHO

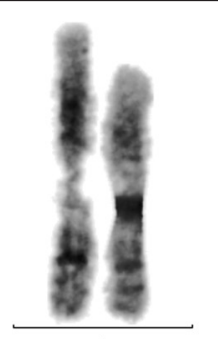

1

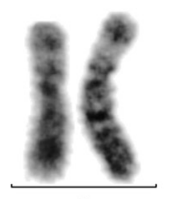

6

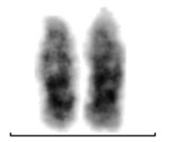

13
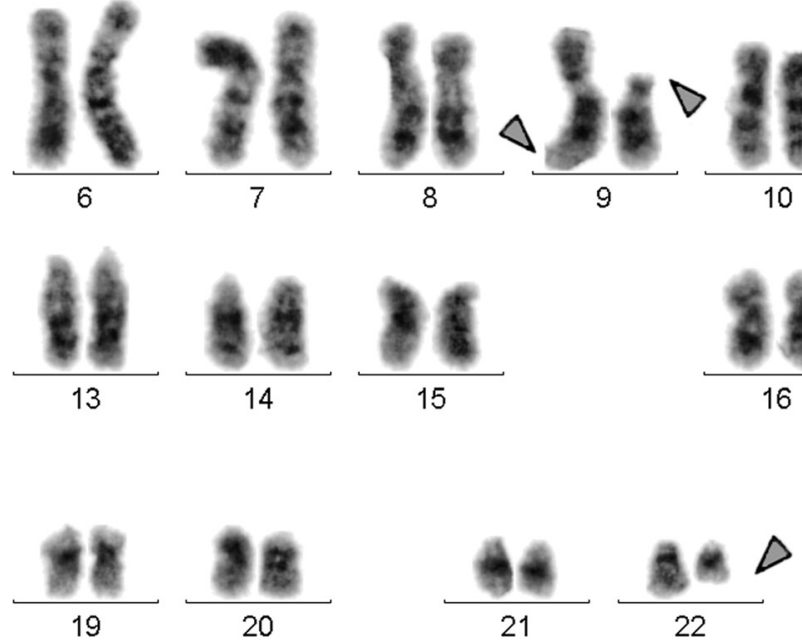

16

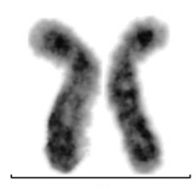

4
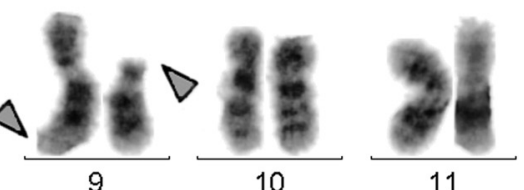

11
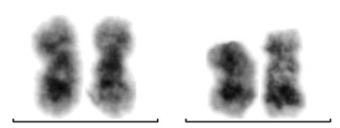

17
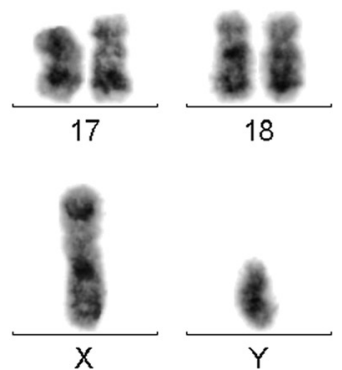

Fig. 1 GTG-banding revealed a deletion of the short arm of a derivative chromosome 9 del(9)(p?). A derivative chromosome is marker by arrowhead 

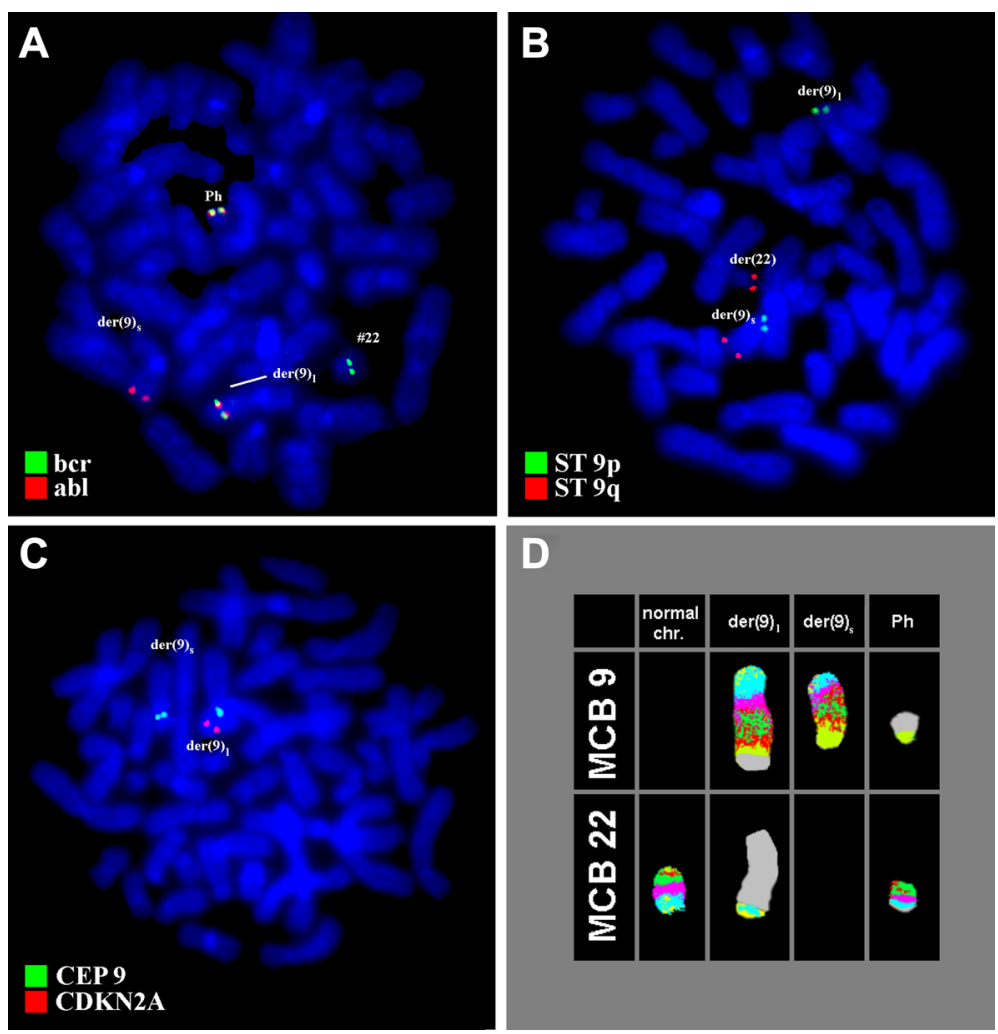

Fig. 2 Karyotype and chromosomal aberrations were confirmed using molecular cytogenetic approaches. a FISH using probes for BCR (green) and ABL (red) showed 2 copies of BCR/ABL on Ph chromosome and on der(9), respectively. b FISH using probes for ST 9p (green) and ST 9q (red) showed; 1 green and 1 red signal on der(9); and 1 red signal on Ph chromosome and 1 green signal on der(9). c The deletion of CDKN2A was identified on the der(9). $\mathbf{d}$ The application of MCB 9 characterized the del(9)(p24p12) comprehensively. Abbreviations: \# = chromosome; der $=$ derivative chromosome

recommendations): $\mathrm{CD} 45^{+\mathrm{dim}}, \mathrm{CD} 13^{+}, \mathrm{CD} 33^{+}, \mathrm{CD} 32^{+}$

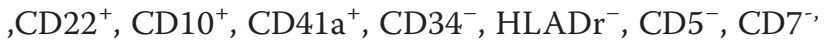

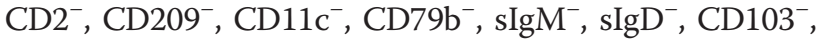
$\mathrm{CD}_{138^{-}}$, CD56 ${ }^{-}, \mathrm{CD}^{-} 7^{-}$and expressed CD64, CD38, and $\mathrm{CD} 4$ heterogeneously.

\section{Conclusions}

We described a de novo untreated CML case in BC with $\operatorname{del}(9)(\mathrm{p} 23 \mathrm{p} 11.1)$ as sole additional acquired abnormality, which leads to monoallely of tumor suppressor gene CDKN2A as located at $9 \mathrm{p} 21$. Up to date, there are 7 CML cases listed in Mitelman Database showed the involvement of the short arm of chromosome 9, two of them revealed involvement of 9p21 [5]. Also, loss of a part of the short arm of chromosome 9 (p12 and/or p21) in CML-BC are reviewed in two previous studies $[5,6]$. To the best of our knowledge, this chromosomal abnormality del(9)(p23p11.1) has not been previously observed in CML [7].

According to the literature, approximately $10 \%$ of CML patients present in advanced phases called accelerated phase (CML-AP) or CML-BC, without a clinically evident CP [8]. Even with the advent of tyrosine kinase inhibitor (TKI) therapy, the phase of disease remains an important prognostic factor in CML $[9,10]$.

The progression to advanced phases of disease is caused by the development of genetic lesions in addition to BCR-ABL1 translocation; secondary cytogenetic changes are identified in more than $80 \%$ of patients by the time of progression to CML-BC [11]. Even in the absence of other features of CML-AP or CML-BC, the acquisition of new cytogenetic abnormalities in patients treated with IM is associated with loss of response and poor prognosis $[12,13]$.

The $\mathrm{t}(9 ; 22)$ or a variant $\mathrm{Ph}$ are frequently the sole chromosomal anomaly during the CP [1]. The most common secondary chromosomal aberrations in advanced phase CML are $+8,+\mathrm{Ph}, \mathrm{i}(17 \mathrm{q}),+19,-\mathrm{Y},+21,+17,-7$, and -17 [1].

The median time to progression from CML-CP to CML-BC is 3-5 years in untreated patients or in patients treated with hydroxyurea [14]. However, BC is typically preceded by an intermediate AP of disease by a period of 4-6 months [15], but up to $25 \%$ of CML-BC cases develop without any clinically evident preceding AP [16]. 
The molecular events associated with CML progression are complex and are likely related to genetic instability and impaired DNA repair mechanisms that are consequences of BCR-ABL1 activity [17-19]. In addition to cytogenetic aberrations, BCR-ABL1 amplification, gene mutations, and epigenetic changes such as gene methylation also appear to play a role in disease progression [20]. Interestingly, different BC phenotypes have been associated with unique patterns of molecular evolution: ABL kinase domain mutations that confer resistance to TKIs occur frequently in lymphoid $\mathrm{BC}$, while myeloid $\mathrm{BC}$ is more commonly associated with cytogenetic evolution. BCR-ABL1 amplification is seen in about $31 \%$ of both myeloid and lymphoid $\mathrm{BC}$ cases [21].

$C D K N 2 A$ and $C D K N 2 B$ are tumor suppressor genes located in 9p21. They belong to the family of inhibitors of cyclin-dependent kinases. The CDKN2A gene encodes two proteins, $\mathrm{p} 14^{\mathrm{ARF}}$ and $\mathrm{p} 16^{\mathrm{INK} 4 \mathrm{a}}$, and the $C D K N 2 B$ gene p15 INK4b protein, which as key regulators of G1 phase cell-cycle arrest and senescence [22]. These genes product is inactivated in a wide range of human cancers through epigenetic mechanisms [22, 23],

Deletion at $9 \mathrm{p} 21$ is especially frequent in acute lymphocytic leukemia (ALL), occurring at more than of $20 \%$ in B-cell precursor ALL and approximately $50 \%$ T-ALL patients [24]. The 9p21 deletions have been suggested to be associated with unfavorable outcome in both adult ALL and pediatric ALL, although the prognostic impact of CDKN2A deletions in pediatric ALL appears controversial [24-27].

Recently, FOXA1 is a key transcription (TF) factor for CDKN2A expression [23, 28]. FOXA1 is a member of forkhead family TFs with remarkable pioneering activity to open closed chromatin for its subsequent cooperation with other master TF in embryogenesis and organ development $[29,30]$.

In conclusion, we described de novo untreated CML case in BC with del(9)(p23p11.1) as sole additional acquired abnormality detected by FISH technique, which leads to monoallely of tumor suppressor gene CDKN2A as located at $9 \mathrm{p} 21$. The patient did not demonstrate a good response to Imatinib treatment.

\section{Materials and Methods}

\section{Chromosome analysis}

Chromosome analysis applying GTG-banding according to standard procedures [31] was performed prior IM treatment. 20 metaphase cells derived from unstimulated bone marrow culture were analyzed. Karyotypes were described according to the International System for Human Cytogenetic Nomenclature (ISCN 2009).

\section{Molecular cytogenetics}

Fluorescence in situ hybridization (FISH) using the LSI $\mathrm{BCR} / \mathrm{ABL}$ dual color dual fusion translocation probe (Abbott Molecular/Vysis, Des Plaines, IL, USA), a locus specific probe for CDKN2A gene (LSI p16 in 9p21) with a probe for centromere nine, and a subtelomeric probe for 9p and 9q (Abbott Molecular/Vysis, Abbott Park, IL, USA) were applied together with whole chromosome painting (WCP) probe for chromosome 9 (MetaSystems, Altlussheim, Germany) according to manufacturer's instructions [31]. Also a multicolor banding probe (MCB) sets based on microdissection derived region-specific libraries for chromosome 9 was applied as previously described [32]. A minimum of ten metaphase spreads was analyzed, using a fluorescence microscope (AxioImager.Z1 mot, Carl Zeiss Ltd., Hertfordshir, UK) equipped with appropriate filter sets to discriminate between a maximum of five fluorochromes plus the counterstain DAPI (4',6- diamino-2-phenylindole). Image capture and processing were performed using an ISIS imaging system (MetaSystems, Altlussheim, Germany).

\section{Reverse transcriptase-polymerase chain reaction (RT-PCR) and for BCR/ABL1 fusion transcripts}

Total RNA extracted from peripheral blood sample using the InviTrap RNA kit (Invitek, Berlin, Germany) according to the manufacturer's recommendations. cDNA was prepared from $5 \mu \mathrm{g}$ of total RNA with the Genequality BCR-ABL1 kit (AB Analitica, Padova, Italy) and BCRABL1 fusion transcript was performed according to the manufacturer's instructions (AB Analitica, Padova, Italy).

\section{Flow cytometric immunophenotype}

Flow cytometry of leukemic blasts was performed using a general panel of fluorescent antibodies against the following antigens typical for different cell lineages and cell types: CD1a, CD2, CD3, CD4, CD5, CD8, CD10, CD11b, CD11c, CD13, CD14, CD15, CD16, CD19, CD20, CD22, CD23, CD32, CD33, CD34, CD38, CD41a, CD45, CD56, CD57, CD64, CD103, CD117, CD123, CD209, CD235a and CD243; in addition antibodies against Kappa and Lambda light Chains, sIgD, sIgM, and HLADr were applied (BD Biosciences). Four-color immunophenotyping on peripheral blood specimen was performed. Samples were stained and analyzed on a BD FACSCalibur ${ }^{\mathrm{TM}}$ flow cytometer according to $\mathrm{BD}$ Biosciences manuals and products insert sheets. Autofluorescence, viability, and isotype controls were included. Flow cytometric data acquisition and analysis were conducted by BD Cellquest ${ }^{\mathrm{TM}}$ Pro software.

\section{Consent}

Written informed consent was obtained from the patient for publication of this Case Report. A copy of the written 
consent is available for review by the Editor-in-Chief of this journal.

\section{Competing interests}

The author(s) declare that they have no competing interests.

\section{Authors' contributions}

AW, MA and FM provided the case and/or did primary cytogenetic and main part of the FISH-tests; AA did the flow cytometry analysis; TL did detailed FISH studies. WA supervised the cytogenetic analysis as Head of HGD. AW and TL drafted the paper and all authors read and approved the final manuscript.

\section{Acknowledgements}

We thank Prof. I. Othman, the Director General of Atomic Energy Commission of SYRIA (AECS) and Dr. N. Mirali, Head of Molecular Biology and Biotechnology Department for their support. This work was supported by the AECS, in parts by the DAAD.

\section{Author details}

'Department of Molecular Biology and Biotechnology, Human Genetics Division, Atomic Energy Commission, P.O. Box 6091, Damascus, Syria.

${ }^{2}$ Department of Molecular Biology and Biotechnology, Mammalians Biology Division, Atomic Energy Commission, Damascus, Syria. ${ }^{3}$ Institute of Human Genetics, Jena University Hospital, Jena, Germany.

Received: 15 April 2015 Accepted: 18 July 2015

Published online: 04 August 2015

\section{References}

1. Johansson B, Fioretos T, Mitelman F. Cytogenetic and molecular genetic evolution of chronic myeloid leukemia. Acta Haematol. 2002;107:76-94.

2. Swerdlow SH, Campo E, Harris NL, Jaffe ES, Pileri SA, Stein H, Thiele J, Vardiman JW. WHO Classification of tumors of haematopoietic andlymphoid tissues. 4th ed. WHO press: IARC; 2008. p. 439.

3. Baccarani M, Deininger MW, Rosti G, Hochhaus A, Soverini S, Apperley JF, et al. European LeukemiaNet recommendationsfor the management of chronic myeloid leukemia: 2013. Blood. 2013;122:871-84.

4. Druker BJ, Guilhot F, O'Brien SG, Gathmann I, Kantarjian H, Gattermann N, et al. Five-year follow-up of patients receiving imatinib for chronic myeloid leukemia. N Engl J Med. 2006;355:2408-17.

5. Mitelman Database of Chromosome Aberrations in Cancer (2015). Mitelman F, Johansson B and Mertens F (Eds.), http://cgap.nci.nih.gov/Chromosomes/ Mitelman" [last accessed 04.02.2015].

6. Pollak C, Hagemeijer A. Abnormalities of the short arm of chromosome 9 with partial loss of material in hematological disorders. Leukemia. 1987;1:541-8.

7. Van PN, Xinh PT, Kano Y, Tokunaga K, Sato Y. Establishment and characterization of A novel Philadelphia-chromosome positive chronic myeloid leukemia cell line, TCC-S, expressing P210 and P190 BCR/ABL transcripts but missing normal ABL gene. Hum Cell. 2005;18:25-33.

8. Jabbour E, Kantarjian H, Jones D, Talpaz M, Bekele N, O'Brien S, et al. Frequency and clinical significance of BCR-ABL mutations in patients with chronic myeloid leukemia treated with imatinib mesylate. Leukemia. 2006;20:1767-73

9. Mitchell B, Deininger M. Techniques for risk stratification of newly diagnosed patients with chronic myeloid leukemia. Leuk Lymphoma. 2011;52:4-11.

10. IRIS Investigators, O'Brien SG, Guilhot F, Larson RA, Gathmann I, Baccarani M, et al. Imatinib compared with interferon and low-dose cytarabine for newly diagnosed chronic-phase chronic myeloid leukemia. N Engl J Med. 2003;348:994-1004

11. Gribble SM, Sinclair PB, Grace C, Green AR, Nacheva EP. Comparative analysis of G-banding, chromosome painting, locusspecific fluorescence in situ hybridization, and comparative genomic hybridization in chronic myeloid leukemia blast crisis. Cancer Genet Cytogenet. 1999;111:7-17.

12. Cortes JE, Talpaz M, Giles F, O'Brien S, Rios MB, Shan J, et al. Prognostic significance of cytogenetic clonal evolution in patients with chronic myelogenous leukemia on imatinib mesylate therapy. Blood. 2003;101:3794-800

13. O'Dwyer ME, Mauro MJ, Blasdel C, Farnsworth M, Kurilik G, Hsieh YC, et al. Clonal evolution and lack of cytogenetic response are adverse prognostic factors for hematologic relapse of chronic phase CML patients treated with imatinib mesylate. Blood. 2004;103:451-5.

14. Sokal JE, Baccarani M, Russo D, Tura S. Staging and prognosis in chronic myelogenous leukemia. Semin Hematol. 1988;25:49-61.

15. Griesshammer M, Heinze B, Hellmann A, Popp C, Anger B, Heil G, et al. Chronic myelogenous leukemia in blast crisis: retrospective analysis of prognostic factors in 90 patients. Ann Hematol. 1996;73:225-30.

16. Kantarjian HM, Deisseroth A, Kurzrock R, Estrov Z, Talpaz M. Chronic myelogenous leukemia: a concise update. Blood. 1993;82:691-703.

17. Melo JV, Barnes DJ. Chronic myeloid leukaemia as a model of disease evolution in human cancer. Nat Rev Cancer. 2007;7:441-53.

18. Salloukh HF, Laneuville P. Increase in mutant frequencies in mice expressing the BCR-ABL activated tyrosine kinase. Leukemia. 2000;14:1401-4.

19. Calabretta B, Perrotti D. The biology of CML blast crisis. Blood. 2004:103:4010-22

20. Asimakopoulos FA, Shteper PJ, Krichevsky S, Fibach E, Polliack A, Rachmilewitz E, et al. ABL1 methylation is a distinct molecular event associated with clonal evolution of chronic myeloid leukemia. Blood. 1999;94:2452-60.

21. Jones D, Luthra R, Cortes J, Thomas D, O'Brien S, Bueso-Ramos C, et al. $B C R-A B L$ fusion transcript types and levels and their interaction with secondary genetic changes in determining the phenotype of Philadelphia chromosome-positive leukemias. Blood. 2008;112:5190-2.

22. Collado M, Blasco MA, Serrano M. Cellular senescence in cancer and aging. Cell. 2007;130:223-33.

23. Zhang $Y$, Tong T. FOXA1 antagonizes EZH2-mediated CDKN2A repression in carcinogenesis. Biochem Biophys Res Commun. 2014;453:172-8.

24. Sulong S, Moorman AV, Irving JA, Strefford JC, Konn ZJ, Case MC, et al. A comprehensive analysis of the CDKN2A gene in childhood acute lymphoblastic leukemia reveals genomic deletion, copy number neutral loss of heterozygosity, and association with specific cytogenetic subgroups. Blood. 2009;113:100-7.

25. Heyman M, Rasool O, Borgonovo Brandter L, Liu Y, Grandér D, Söderhäll S, et al. Prognostic importance of p15INK4B and p16INK4 gene inactivation in childhood acute lymphocytic leukemia. J Clin Oncol. 1996;14:1512-20.

26. Kees UR, Burton PR, Lu C, Baker DL. Homozygous deletion of the p16/MTS1 gene in pediatric acute lymphoblastic leukemia is associated with unfavorable clinical outcome. Blood. 1997;89:4161-6.

27. Heerema NA, Sather HN, Sensel MG, Liu-Mares W, Lange BJ, Bostrom BC, et al. Association of chromosome arm $9 p$ abnormalities with adverse risk in childhood acute lymphoblastic leukemia: a report from the Children's Cancer Group. Blood. 1999:94:1537-44.

28. Li Q, Zhang Y, Fu J, Han L, Xue L, Lv C, et al. FOXA1 mediates p16(INK4a) activation during cellular senescence. EMBO J. 2013;32:858-73.

29. Jozwik KM, Carroll JS. Pioneer factors in hormone-dependent cancers. Nat Rev Cancer. 2012:12:381-5.

30. Katoh M, Igarashi M, Fukuda H, Nakagama H, Katoh M. Cancer genetics andgenomics of human FOX family genes. Cancer Lett. 2013;328:198-206.

31. AL-achkar W, Nweder MS WA. A complex translocation $\mathrm{t}(5 ; 9 ; 22)$ in Philadelphia cells involving the short arm of chromosome 5 in a case of chronic myelogenous leukemia. J Exp Clin Cancer Res. 2007;26:411-5.

32. Liehr T, Heller A, Starke H, Rubtsov N, Trifonov V, Mrasek K, et al. Microdissection based high resolution multicolor banding for all 24 human chromosomes. Int J Mol Med. 2002;9:335-9.

\section{Submit your next manuscript to BioMed Central and take full advantage of:}

- Convenient online submission

- Thorough peer review

- No space constraints or color figure charges

- Immediate publication on acceptance

- Inclusion in PubMed, CAS, Scopus and Google Scholar

- Research which is freely available for redistribution 